University of Nebraska - Lincoln

DigitalCommons@University of Nebraska - Lincoln

John R. Hardy Papers

Research Papers in Physics and Astronomy

6-1-1985

\title{
First-Principles Study of Structural Instabilities in Halide-Based Perovskites: Competition Between Ferroelectricity and Ferroelasticity
}

\author{
J. W. Flocken \\ University of Nebraska at Omaha \\ R. A. Guenther \\ University of Nebraska at Omaha \\ John R. Hardy \\ University of Nebraska - Lincoln \\ L. L. Boyer \\ U. S. Naval Research Laboratory, Washington, D.C.
}

Follow this and additional works at: https://digitalcommons.unl.edu/physicshardy

Part of the Physics Commons

Flocken, J. W.; Guenther, R. A.; Hardy, John R.; and Boyer, L. L., "First-Principles Study of Structural Instabilities in Halide-Based Perovskites: Competition Between Ferroelectricity and Ferroelasticity" (1985). John R. Hardy Papers. 37.

https://digitalcommons.unl.edu/physicshardy/37

This Article is brought to you for free and open access by the Research Papers in Physics and Astronomy at DigitalCommons@University of Nebraska - Lincoln. It has been accepted for inclusion in John R. Hardy Papers by an authorized administrator of DigitalCommons@University of Nebraska - Lincoln. 


\title{
First-principles study of structural instabilities in halide-based perovskites: Competition between ferroelectricity and ferroelasticity
}

\author{
J. W. Flocken and R. A. Guenther \\ University of Nebraska-Omaha, Omaha, Nebraska 68101 \\ J. R. Hardy \\ University of Nebraska-Lincoln, Lincoln, Nebraska 68588-0111 \\ L. L. Boyer \\ U. S. Naval Research Laboratory, Washington, D.C. 20375 \\ (Received 8 January 1985)
}

\begin{abstract}
We have made a systematic theoretical survey of the competition between ferroelastic and ferroelectric instabilities in the family of halide-based perovskites of formula $A B X_{3}$, where $A$ is an alkali-metal ion, $B$ is a $\mathrm{Be}, \mathrm{Mg}$, or $\mathrm{Ca}$ ion, and $X$ is a halide ion. Initially we surveyed the whole series of such compounds, making a theoretical lattice-dynamical study using first-principles interionic potentials composed of a long-range pure Coulomb interaction between the spherically symmetric free ions, and a short-range component calculated by the Gordon-Kim approach from the overlapping free-ion charge densities. We then proceeded to examine in more detail three compounds, $\mathrm{NaCaBr}_{3}, \mathrm{NaCaCl}_{3}$, and $\mathrm{NaCaF}_{3}$, which manifested both ferroelectric (zone-center) and ferroelastic (zone-boundary) instabilities (there were no structures which showed zone-center instabilities alone). For these three systems we then proceeded to a full energy minimization. This was done by allowing all 40 ions in the lowest symmetry phase to relax independently. It was found that the most stable structure of all three compounds consisted of triply canted halide octahedra, turned through equal angles about all three Cartesian axes. In this phase all eigenfrequencies are real, implying absolute stability, and the ferroelectric instability has been removed. We also discuss the possibility of a ferroelectric, or near-ferroelectric, intermediate phase.
\end{abstract}

\section{INTRODUCTION}

Probably the two most studied structural phase transitions are the ferroelectric transition at $393 \mathrm{~K}$ in barium titanate $\left(\mathrm{BaTiO}_{3}\right)$ and the ferroelastic transition at $105 \mathrm{~K}$ in strontium titanate $\left(\mathrm{SrTiO}_{3}\right)$, which arises from instability of a triply degenerate mode at the Brillouin-zone corner. At present these and similar instabilities in chalcogenbased systems are not amenable to first-principles theoretical study, principally because free chalcogen ions, at least in the doubly negative state, are unstable. Thus one lacks even a zero-order approximation to the crystalline charge density. Recently, however, a body of work $^{1-8}$ has demonstrated that the fluorine-based halide perovskites, such as $\mathrm{RbCaF}_{3}$ (the most extensively studied), also display zone-boundary instabilities. Subsequently, in several papers, ${ }^{9-13}$ we have demonstrated that it is possible to explain theoretically, from first principles, both qualitatively and quantitatively, the origins of these and other classes of instability. At present this work is confined to halide-based systems, since it requires a reliable knowledge of the electronic charge density in the crystal which, in halide perovskites, can be taken as superposition of the free-ion charge densities, since the ions are stable and their overlap in the crystal is small.

In the light of this success for $\mathrm{RbCaF}_{3}$, it was a logical next step to carry out a systematic study of virtually all of the halide-based perovskites to determine which of them were stable, which were unstable, and the nature of the instabilities. Apart from its inherent scientific value, there is also the practical virtue of this type of structural analysis, since one can study the whole sequence in a fraction of the time, and at an even smaller fraction of the cost, of chemically and physically synthesizing just one member. We also had one specific subobjective, which was to determine whether some member(s) of the sequence were the analogs of $\mathrm{BaTiO}_{3}$ in displaying ferroelectric behavior.

To avoid an inordinate expenditure of time we chose to examine only the static energies, deferring for possible future work free-energy calculations of the type carried out ${ }^{11}$ for $\mathrm{RbCaF}_{3}$. This last work indicated that the present approach could provide a reliable "zero-order" profile of these systems and should certainly reveal potential ferroelectricity which could, if found, be further studied by a full free-energy calculation, to determine whether the ferroelectric state was stable in the presence of thermal and zero-point motion.

Insofar as we have, thus far, been unable to establish the possibility of ferroelectric behavior in halide perovskites, it would appear that there may be a special association of ferroelectricity with chalcogen-based systems. Moreover, the present treatment, which regards the ions as rigid and unaffected by the crystal environment, is certainly not fully adequate to handle chalcogen ions which, in the doubly negative state, are unstable when 
free. Thus, there may well be new effects, analogous to those recently reported for simple oxides, ${ }^{14}$ associated with ionic self-energy changes induced by lattice distortions. However, the incipient ferroelectric distortion we find always appears in conjunction with a strong zoneboundary instability. There appears to be a good qualitative reason for this. The most likely halide perovskites to show ferroelectric behavior are those having the smallest divalent cations at the centers of the halogen-ion octahedra since, if this ion is sufficiently small, the "size" of this octahedron will be determined by halogen-halogen contact, and the divalent cation will be essentially unconstrained to remain on site. However, should the monovalent cation also be small, the halide octahedra will tend to rotate, since they can thus pack more closely and reduce the lattice energy. It appears, except possibly in a few extreme cases involving ions with very few electrons (for which our potentials are least reliable), that the relative ion sizes are such that the zone-boundary instability is always strongly dominant (how it suppresses the ferroelectric instability is less obvious). Thus, before we invoke an entirely novel mechanism for the behavior of chalcogenbased perovskites, it is worth asking if it is not explicable, at least in part, by ionic size effects which tend to weaken the $R$-point instability and favor ferroelectricity.

Examination of the Pauling nominal ionic radii ${ }^{15}$ for $\mathrm{Ba}^{2+}, \mathrm{Sr}^{2+}, \mathrm{Ti}^{4+}$, and $\mathrm{O}^{2-}$, suggests that the presence of an $R$-point zone-boundary instability in $\mathrm{SrTiO}_{3}$ and its absence in $\mathrm{BaTiO}_{3}$ may well be governed by such considerations. How the substitution of $\mathrm{Sr}^{2+}$ for $\mathrm{Ba}^{2+}$ hardens the zone-center instability is less apparent. That the overall force balance in chalcogen-based perovskites is very delicate is further evidenced by the fact that the halide octahedra rotations observed for fluoperovskites (the only family to be extensively studied experimentally) are an order of magnitude larger than those in $\mathrm{SrTiO}_{3}$; an indication that the substitution of $\mathrm{Sr}^{2+}$ or $\mathrm{Ba}^{2+}$ is a much more subtle change than, for example, a $\mathrm{Cs}^{+}$for $\mathrm{Rb}^{+}$ substitution.

Before we proceed to a detailed description of our work, a caveat is in order. As we model the crystal in the computer, we take as our initial configuration the ideal perovskite structure and, insofar as we find it to be unstable, it is with respect to relatively small, although well beyond harmonic, distortions. We cannot rule out the possibility that truly radical ionic rearrangements will produce a structure of lower energy which could have completely different symmetry and coordination. ${ }^{16}$

Our studies are concerned with the halide perovskites of general formula $A B X_{3}$, where $A$ is an alkali-metal ion, $B$ is an alkaline-earth ion, and $X$ is a halide ion. The unit cell for this structure is shown in Fig. 1. 60 compounds of this type were selected for the initial investigation. These were comprised of all possible combinations of the alkali-metal ions $\mathrm{Li}^{+}, \mathrm{Na}^{+}, \mathrm{K}^{+}, \mathrm{Rb}^{+}$, and $\mathrm{Cs}^{+}$, the alkaline-earth ions $\mathrm{Be}^{2+}, \mathrm{Mg}^{2+}$, and $\mathrm{Ca}^{2+}$, and the halide ions $\mathrm{F}^{-}, \mathrm{Cl}^{-}, \mathrm{Br}^{-}$, and $\mathrm{I}^{-}$. The theoretical approach used in these calculations was similar to that used by Boyer and Hardy ${ }^{11}$ in their study of structural phase transitions in $\mathrm{RbCaF}_{3}$, and will be discussed more fully in the following section. Since we are interested in seeing

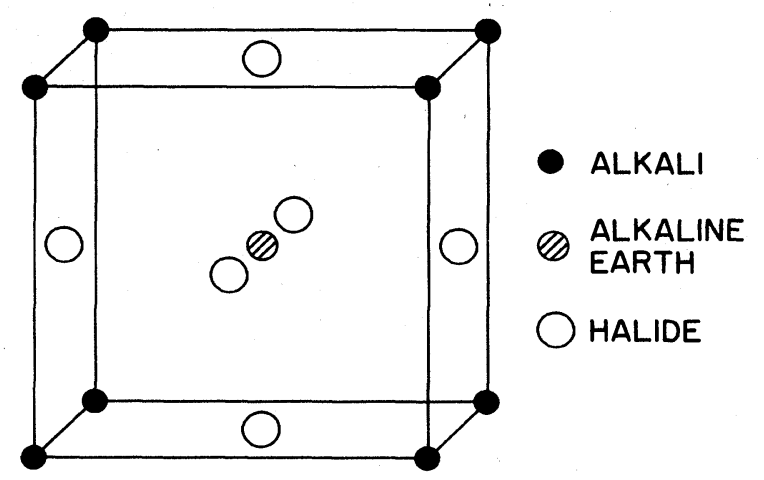

FIG. 1. Unit cell of the perovskite structure.

whether these materials are stable in the perovskite configuration and specifically if they might exhibit ferroelectric properties, we were looking for an instability at the zone center only, or an instability at the zone center which was more dominant than instabilities at any other points on the boundary of the Brillouin zone. Of the compounds that exhibited such instabilities, three were selected for a more extensive investigation: $\mathrm{NaCaBr}_{3}, \mathrm{NaCaCl}_{3}$, and $\mathrm{Na}$ $\mathrm{CaF}_{3}$. The phase transitions in these three compounds were investigated by allowing the ions in a unit cell of the lattice to relax until the forces on them and the lattice stresses were zeroed, the static energy was minimized, and all soft-mode frequencies became real.

In the following section we will discuss the approach used in calculating the interionic potentials and studying the lattice dynamics of each crystal. In Sec. III we will discuss the general results for the 60 compounds studied. Section IV will be devoted to a description of the techniques used during the in-depth study of the three materials mentioned previously, and the last section will discuss the results of these investigations.

\section{COMPUTATIONAL TECHNIQUES}

The short-range interionic potentials for each ion pair in the crystal under investigation were computed using the Gordon-Kim ${ }^{17}$ approach. This procedure treats each ion in the structure as a closed-shell system for which the electronic charge distribution is computed from the Slater orbital coefficients tabulated by Clementi and Roetti. ${ }^{18}$ The net charge distribution for a pair of ions at a given interionic separation is then assumed to be the sum of the individual ionic charge distributions. The changes in kinetic energy, Coulomb potential, exchange energy, and correlation, due to ionic overlap, are then computed from these charge distributions. These various contributions to the short-range potentials were computed over an appropriate range of interionic separations for each set of ion pairs in the crystal and each contribution was then fitted to an exponential form

$$
U=\exp \left(\alpha+\beta r+\gamma r^{2}\right)
$$

where $r$ is the interionic spacing and $\alpha, \beta$, and $\gamma$ are constants determined in the fitting process. Using these po- 
tentials and the long-range Coulomb potentials calculated by the summation procedure of Ewald, ${ }^{19}$ the dynamical matrix elements of the crystal were computed and the eigenvalues obtained for a regular sample of wave vectors. The total static energy for the perovskite structure was also calculated in each case. In these calculations firstneighbor alkali-metal-ion-halide-ion interactions and alkaline-earth-ion-halide-ion interactions were used. Halide-ion-halide-ion interactions out to third nearest neighbors were retained. Interactions between positive-ion pairs were considered to be negligible. Such calculations were performed for a range of lattice constants for each material, and the results were examined to determine the lattice constant that gave the lowest static energy configuration. The eigenfrequencies for these lowest-energy configurations were then examined to determine whether any instabilities existed.

\section{RESULTS OF THE PRELIMINARY INVESTIGATION}

The lattice constants, static energy per unit cell and imaginary frequencies, if any, for the 60 compounds originally studied are shown in Tables I through III. Table I contains the results for crystals containing beryllium as the alkaline-earth ion. An examination of this table shows a consistent increase in lattice parameter with increasing size of the alkali-metal ion or the halide ion, as one would expect, along with a corresponding increase, in most cases, of the static energy (less negative). The latter is presumably mainly due to a decrease in the negative Madelung energy with increasing lattice constant. It can also be seen that approximately one third of these crystals do not exhibit any instabilities at the equilibrium value of the lattice constant. Of the remainder, only compounds

TABLE I. $A \mathrm{Be} X_{3}$ compounds. $a$ is the lattice constant for minimum static energy (in bohr), $E$ is the energy per unit cell (in $\mathrm{eV}$ ), and $\omega$ is the frequency (in $\mathrm{cm}^{-1}$ ) (only negative, i.e., imaginary, values are shown), together with symmetry point and multiplicity, e.g., $D M$ means a doublet at the $M$ point.

\begin{tabular}{|c|c|c|c|c|c|c|c|c|c|}
\hline & & & $\mathrm{F}$ & & $\mathrm{Cl}$ & & $\mathrm{Br}$ & & I \\
\hline \multirow[t]{9}{*}{$\mathrm{Li}$} & $a$ & & 6.58 & & 8.17 & & 8.86 & & 9.40 \\
\hline & $E$ & & -44.97 & & -37.07 & & -34.76 & & -33.24 \\
\hline & $\omega$ & $D \Gamma$ & -160 & $D \Gamma$ & -83.8 & $D \Gamma$ & -85.0 & $D \Gamma$ & -38.0 \\
\hline & & $T R$ & -161 & $T R$ & -98.0 & $T R$ & -62.0 & $T R$ & -47.6 \\
\hline & & $D X$ & -109 & $D X$ & -49.8 & $D X$ & -53.3 & $S M$ & -47.0 \\
\hline & & $S M$ & -159 & $S M$ & -96.6 & $D M$ & -61.8 & $D M$ & -29.6 \\
\hline & & $D M$ & -123 & $D M$ & -58.8 & $S M$ & -61.3 & & \\
\hline & & $S M$ & -55.0 & & & & & & \\
\hline & & & . & & & & & & \\
\hline \multirow[t]{4}{*}{$\mathrm{Na}$} & $a$ & & 6.74 & & 8.35 & & 8.94 & & 9.49 \\
\hline & $E$ & & -44.18 & & -36.59 & & -34.41 & & -32.87 \\
\hline & $\omega$ & & & $T R$ & -37.6 & $T R$ & -34.2 & $T R$ & -25.0 \\
\hline & & & & $S M$ & -34.3 & $S M$ & -32.8 & $S M$ & -24.0 \\
\hline \multirow[t]{7}{*}{$\mathbf{K}$} & $a$ & & 7.00 & & 8.46 & & 9.04 & & 9.58 \\
\hline & $E$ & & -43.19 & & -36.34 & & -34.32 & & -32.84 \\
\hline & $\omega$ & $D \Gamma$ & -172 & & & & & & \\
\hline & & $D R$ & -154 & & & & & & \\
\hline & & $D X$ & -167 & & & & & & \\
\hline & & $S M$ & -172 & & & & & & \\
\hline & & $S M$ & -152 & & & & & & \\
\hline \multirow[t]{7}{*}{$\mathbf{R b}$} & $a$ & & 7.21 & & 8.60 & & 9.17 & & 9.72 \\
\hline & $E$ & & -42.22 & & -35.95 & & -34.03 & & -32.58 \\
\hline & $\omega$ & $D \Gamma$ & -276 & & & & & & \\
\hline & & $D R$ & -215 & & & & & & \\
\hline & & $D X$ & -277 & & & & & & \\
\hline & & $S M$ & -280 & & & & & & \\
\hline & & $S M$ & -214 & & & & & & \\
\hline \multirow[t]{7}{*}{ Cs } & $a$ & & 7.45 & & 8.74 & & 9.26 & & 9.78 \\
\hline & $E$ & & -41.20 & & -35.73 & & -34.00 & & -32.68 \\
\hline & $\omega$ & $D \Gamma$ & -378 & & & & & & \\
\hline & & $D R$ & -269 & & & & & & \\
\hline & & $D X$ & -376 & & & & & & \\
\hline & & $S M$ & -377 & & & & & & \\
\hline & & $S M$ & -268 & & & & & & \\
\hline
\end{tabular}


incorporating lithium or fluorine as a component have dominant zone-center instabilities and in every case there also are competing instabilities at the " $R$ point" (the corner of the Brillouin zone), and other symmetry points. In the case of the $\mathrm{Li}^{+}$compounds, the $R$-point instabilities are triply degenerate, and are associated with rotations of the halide octahedra about the three coordinate axes of the cell, resulting in a ferroelastic transition. The doubly degenerate $R$-point instabilities also exhibited by the potassium rubidium and cesium compounds must be associated with some other type of displacement.

Because $\mathrm{Li}^{+}$and $\mathrm{Be}^{2+}$ are both very small ions, each retaining only two electrons in the ionic state, it is perhaps valid to object that the Gordon-Kim approach with its free-electron local-density functions may not produce interionic potentials which are as reliable as those for heavier ions. Predominantly for this reason no crystals containing either or both were selected for more extensive study.

An examination of Table II for the $\mathrm{AMgX}_{3}$ compounds shows similar trends, except that zone-center instabilities are now present only for the lithium compounds and $\mathrm{NaMgBr}$. The former we have already excluded; for the present we also exclude the latter because of the large disparity of size between $\mathrm{Mg}^{2+}$ and $\mathrm{Br}^{-}$. In all cases, the zone-boundary instabilities are dominant. Table III, which contains results for the calcium compounds, shows that many of these exhibit instabilities which are considerably more complicated than those seen in the previous tables. However, only the lithium and sodium compounds appear to be likely candidates for a ferroelectric transition since, for those materials, the zone-center instability clearly competes with those at the zone boundary. However, since we have already excluded lithium compounds from consideration, it appears that the only candidates for further investigation are $\mathrm{NaCaF}_{3}, \mathrm{NaCaCl}_{3}$, and $\mathrm{NaCaBr}_{3}$.

\section{COMPUTATIONAL METHODS USED IN THE EXTENDED STUDY}

Since only this small number of the halide perovskites seemed to be possible candidates to display a ferroelectric transition, more extensive calculations were performed for all of them, starting with $\mathrm{NaCaBr}_{3}$. For this material, programs were developed to calculate forces, stresses, and static energies for a lattice in which the ions were dis-

TABLE II. $A \mathrm{Mg} X_{3}$ compounds. $a$ is the lattice constant for minimum static energy (in bohr), $E$ is the energy per unit cell (in $\mathrm{eV}$ ), $\omega$ is the frequency (in $\mathrm{cm}^{-1}$ ) (only negative, i.e., imaginary, values are shown).

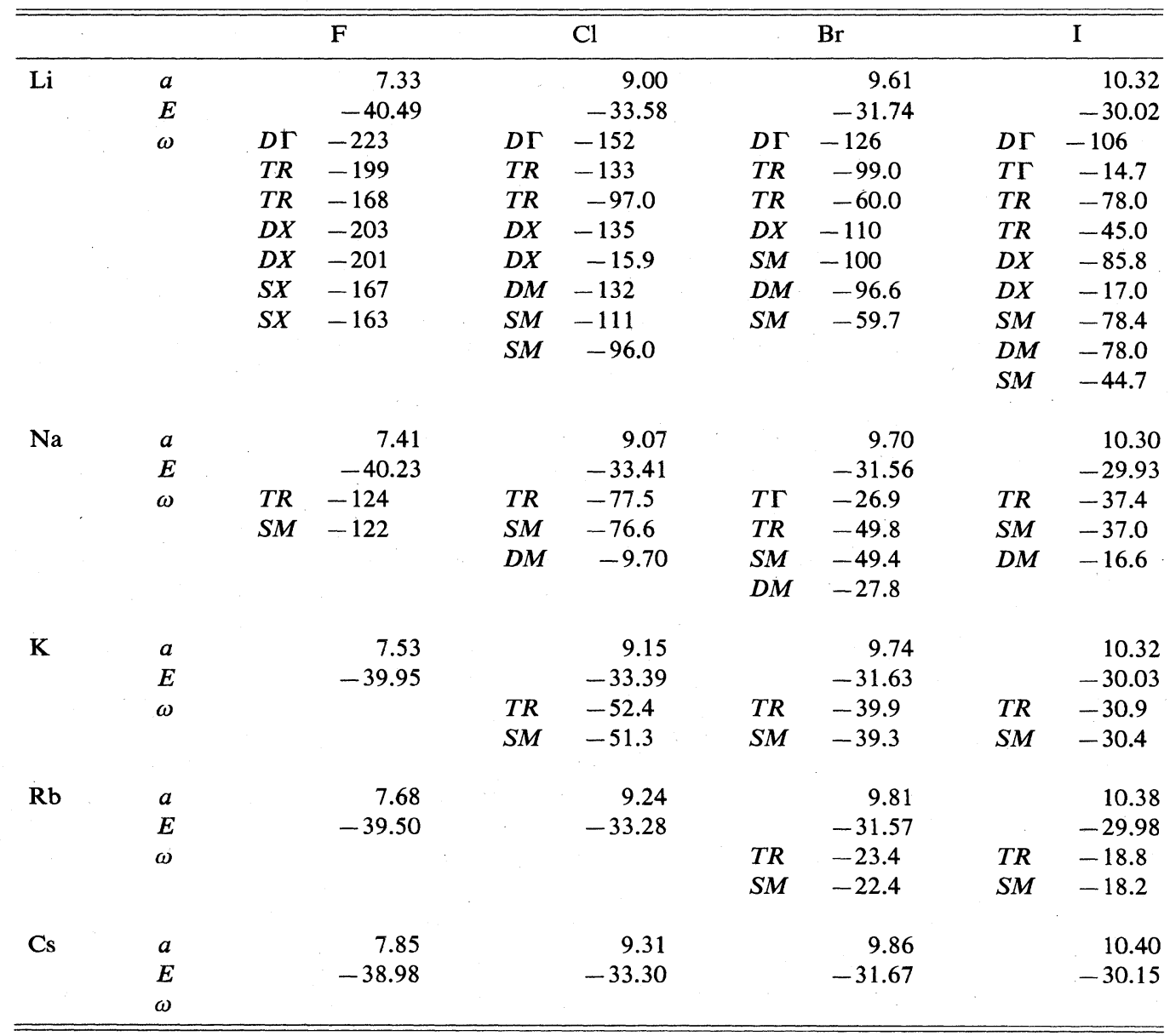


TABLE III. $A \mathrm{CaX}$ compounds. $a$ is the lattice constant for minimum static energy (in bohr), $E$ is the energy per unit cell (in $\mathrm{eV}$ ), $\omega$ is the frequency (in $\mathrm{cm}^{-1}$ ) (only negative, i.e., imaginary, values are shown).

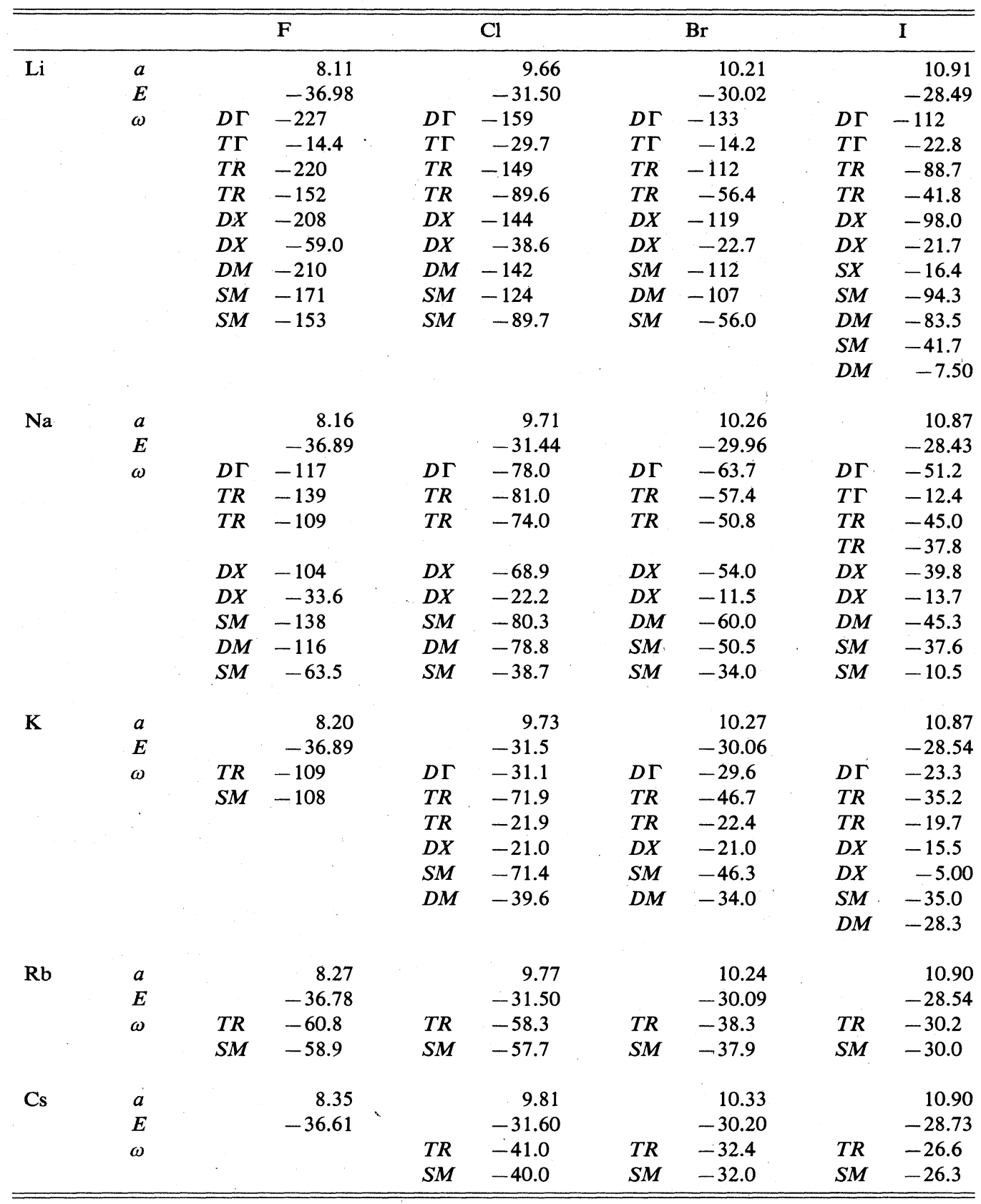

placed from their positions in the high-symmetry (perfect perovskite) structure. This allowed us to determine the lowest-energy configuration for various types of displacive transitions. In addition, the eigenvalue program was modified to allow the calculation of eigenvalues and eigenvectors for the lower-symmetry configurations. Initially this allowed us to determine the types of translations and/or rotations associated with the various "soft modes" which had been found during the initial study and to attempt to "freeze" these in by imposing permanent displacements of the required type of the ions within a suitably chosen unit cell. These calculations were useful in that we were able to "track" the instabilities at the zone center and the $R$ point. As one might expect, the zonecenter soft mode was found to be stabilized by purely translational displacements along the $x$ or $y$ directions when the small but finite wave vector employed was along the $z$ axis, i.e., by transverse-optic motions, and the $R$ point instability was found to be associated with a rotation of the bromine octahedra about the $x, y$, or $z$ axes. 
Ultimately, however, in order to determine the lowestenergy configuration which stabilized the lattice completely, it was necessary to generalize the static energy and the eigenvalue programs to handle any desired configuration of ions in the unit cell. Using this generalized static program, all of the ions in the unit cell were allowed to relax to positions which zeroed the forces on each ion. In order to obtain zero net normal and shear stresses it was also necessary to allow the lattice parameter to vary, and to allow the coordinate axes to shear from the original orthogonal coordinate system. The zeroing process was accomplished by an iterative calculation in which the coordinates of each ion were varied by an amount proportional to the net force on that ion, while the lattice constant and the angles between the old coordinate axes and the new nonorthogonal axes, were varied by amounts proportional to the net normal and shear stresses, respectively. The iterations were continued until the forces and stresses were reduced to within a specified tolerance level. At this point the eigenvalue program was used to determine whether the eigenfrequencies for this configuration were all real and, if such were the case, the net dipole moment per unit volume was calculated. In carrying out these calculations it was necessary, in order to preserve translational symmetry, to choose a unit cell for the lowsymmetry structure which consisted of eight of the highsymmetry until cells; i.e., the lattice parameter along each of three coordinate axes was twice that for the unit cell of the perovskite structure. Thus in the low-symmetry configuration there were 40 ions per unit cell rather than five.

\section{RESULTS OF THE EXTENDED STUDY}

The codes described above were used initially to determine the eigenvectors associated with the instability at the zone center, which permitted us to find the relative displacements of the $A, B$, and $X$ ions for this normal mode. These ions were then allowed to relax, keeping their relative displacements constant, and the static energy of the unit cell was calculated as a function of these displacements. The resulting potential well for the case of $\mathrm{NaCaBr}_{3}$ is shown in Fig. 2, and the displacements which result in a minimum energy are listed in Table IV. A similar calculation was carried out for the $R$-point insta-

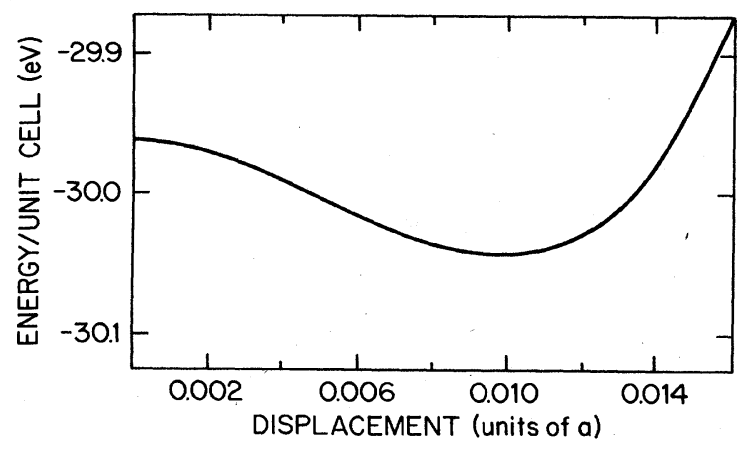

FIG. 2. Plot of the energy versus bromine displacement for the zone-center instability in $\mathrm{NaCaBr}_{3}$.

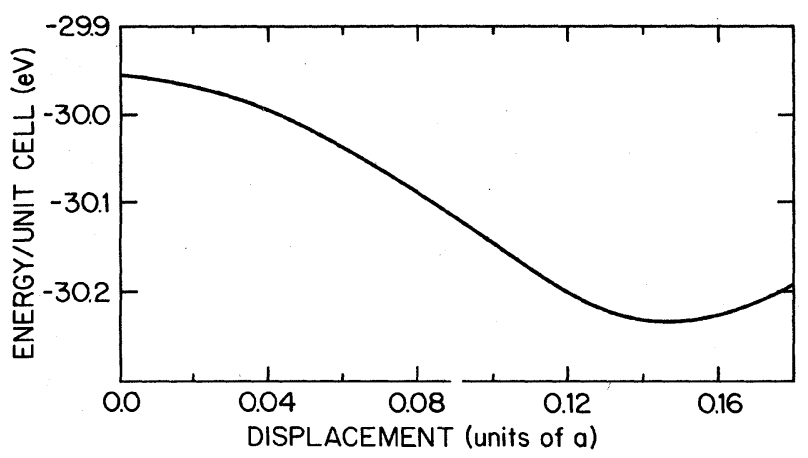

FIG. 3. Plot of energy versus bromine displacement for the zone-corner ( $R$-point) instability for $\mathrm{NaCaBr}_{3}$.

bility. For that case, the static energy per unit cell was computed as a function of "rotation" angle for a single rotation of the bromine ions about the $z$ axis of the cell. A plot of this potential well is shown in Fig. 3. The minimum occurs for a rotation of about $18^{\circ}$. It should be pointed out that we are not performing "pure" rotations, as the bromine ions are constrained by symmetry to remain in the $x-y$ planes of the cell sides in which they were originally located. It was found that such a rotation did, in fact, remove one of the imaginary frequencies at the $R$ point, but did not stabilize the original zone-center instability, also new instabilities appeared at the zone center associated with the "folding back" of the remaining unstable $R$-point doublet. Similarly, purely translational displacements did not remove the $R$-point instabilities, although the threefold degeneracy at the $R$ point was broken. Table $\mathrm{V}$ shows the static energies per unit cell of the pure perovskite structure at the minima of the wells produced by the separate rotational and translational displacements. As is readily apparent, the rotational displacements result in a much lower energy than the translational displacements. It should be noted that, while it was necessary in performing the constrained minimization for the rotation to use a unit cell which consisted of four unit cells of the high-symmetry structure, the energies quoted are in terms of the original unit cell of five ions.

At this point the eigenvectors for the zone-center soft mode were recomputed with the rotational displacement frozen-in and a calculation was done in which both rotational and translational displacements were permitted. The result of this calculation is also shown in Table $\mathrm{V}$. The energy minimum in this case occurred for a rotation of about $16^{\circ}$, with very small translations of the ions in the cell, but the static energy did not decrease significant-

TABLE IV. Translational displacements for $\mathrm{NaCaBr}_{3}$ at minimum static energy (along $x$ axis, in bohr).

\begin{tabular}{lr}
\hline \hline $\mathrm{Na}$ & 1.65 \\
$\mathrm{Ca}$ & 0.213 \\
$\mathrm{Br}(x z$ and $y z$ planes $)$ & -0.332 \\
$\mathrm{Br}(y z$ plane $)$ & 0.103 \\
\hline
\end{tabular}


TABLE V. Total static energy per unit cell (in $\mathrm{eV}$ ) for $\mathrm{NaCaBr}_{3}$ (see also Table VI).

\begin{tabular}{ll}
\hline \hline & \\
\hline Perovskite structure & -29.9640 \\
x-axis translation & -30.0458 \\
Rotation about $z$ axis $\left(18.3^{\circ}\right)$ & -30.2343 \\
Translation plus $16^{\circ}$ rotation & -30.2370 \\
General displacement & -30.487 \\
\hline \hline
\end{tabular}

ly from that found for the pure rotation instability.

In order to find the configuration which would give an "absolute" minimization of the static energy, the lattice parameter and ionic positions were permitted to relax without constraint until the normal stresses and the net force on each ion were zeroed. In addition, in order to zero the shear stresses, a nonorthogonal lattice was generated by allowing the original crystal axes to rotate through a small angle with respect to their original directions. The stresses imposed by the displacements of the ions had identical, $x, y$, and $z$ coordinates, hence the lattice parameter remained the same along each of the three axes and the "shear angle" was also the same for each of the new axes relative to the original ones. The resultant structure is schematically illustrated in Fig. 4 and the actual parameters which resulted in a minimum energy configuration are shown in Table VI, along with the static energy, which is also listed in Table $\mathrm{V}$. This energy is considerably lower than those obtained from the previous calculations, showing a decrease of $0.52 \mathrm{eV}$ relative to the static energy per unit cell of the undistorted perovskite phase. A run of the eigenfrequency program showed that this configuration stabilizes all of the soft modes found originally. However, the ionic displacements give no net dipole moment.

"Generalized displacement" calculations of the type just described were also carried out for $\mathrm{NaCaCl}_{3}$ and $\mathrm{NaCaF}_{3}$, with results which were very similar to those obtained for $\mathrm{NaCaBr}_{3}$. The parameters for each which minimize the energy and stabilize the lattice are also shown in Table VI, together with the static energies for the highand low-symmetry structures. It would appear from our investigation of these three halide-based perovskites that the energies of the low-symmetry structures are sufficiently far below those of the perovskite phase for these materials to exist only in this low-symmetry configuration, ${ }^{20}$ since the transition to the high-symmetry structure may occur above their melting points.

\section{CONCLUSIONS}

We have made a systematic theoretical investigation of the family of halide perovskites containing monovalent and divalent cations from the first two columns of the Periodic Table. While we find that many of them show ferroelastic instabilities associated with $R$-point (zonecorner) phonons, it appears that when there is a $\Gamma$-point (zone-center) instability in the perovskite phase, this disappears in the distorted structure produced by freezing-in the $R$-point instabilities. Thus the resultant

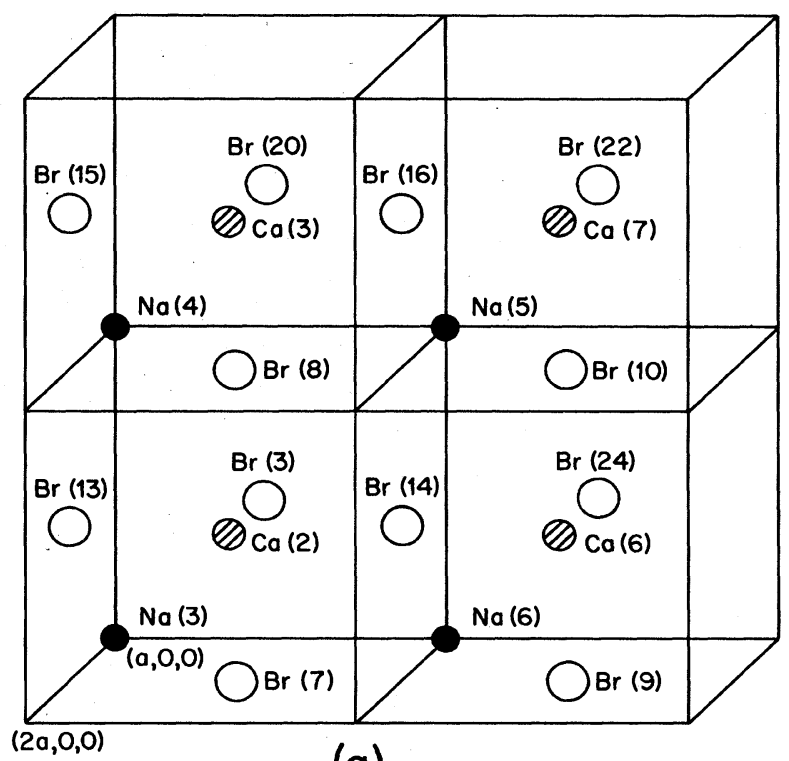

(a)

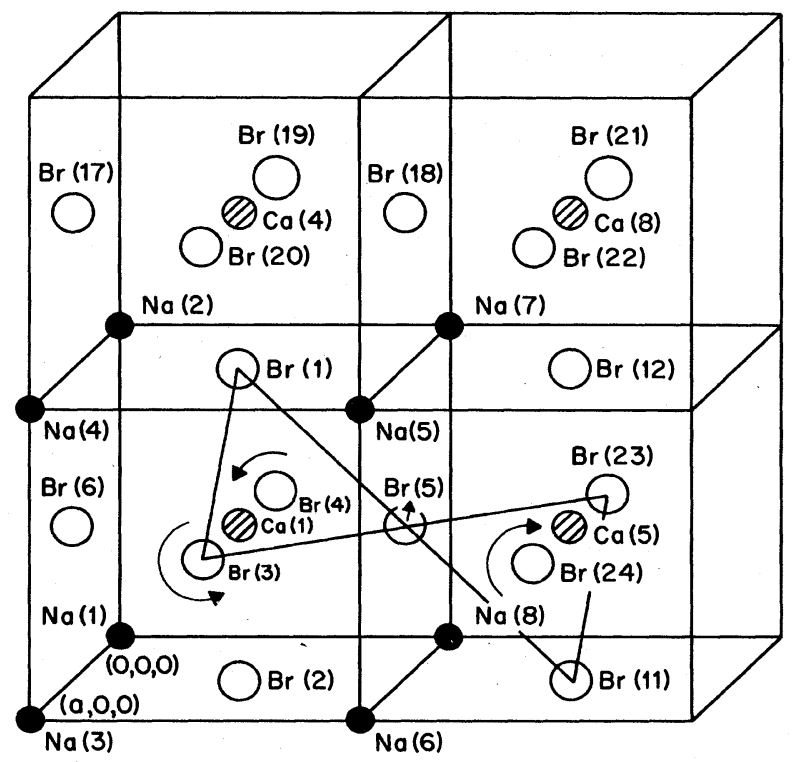

(b)

FIG. 4. Schematic illustration of the low-symmetry structure and its relation to the original perovskite structure: (a) front four cells, (b) rear four cells. The ions are identified by number for reference to Table VI to determine their actual displacements.

structures consist of phased, triply canted octahedra (equal rotations about all three $\langle 100\rangle$ directions). In this structure there is no polar distortion.

We finally return to our subsidiary objective of determining whether or not there is something peculiar to the chalcogen ions that makes them particularly able to produce ferroelectricity. It would appear that the answer is a qualified affirmative. Such tendencies to ferroelectric behavior as we have found appear to be the exception rather than the rule. Where they exist they appear to be 
TABLE VI. Lattice constants, displacements, and energy/cell for fully relaxed $\mathrm{NaCa} X_{3}$ structures. $a$ is the lattice constant, and $\theta$ is the shear angle. Displacements are given prior to shearing in units of $2 a$ in order to show the symmetry. The energies are given for the fully relaxed system.

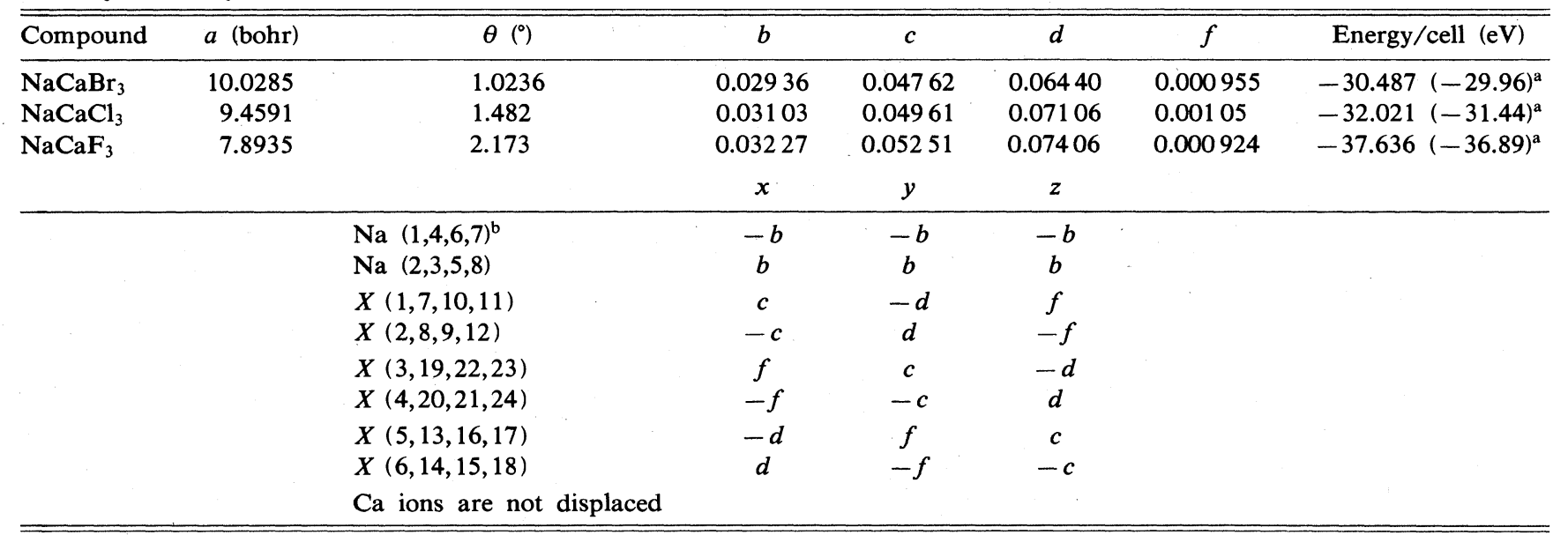

${ }^{a}$ Corresponding energies for the perovskite phase.

${ }^{b}$ Refer to Figs. 4(a) and 4(b) for ionic indices.

invariably suppressed by a dominant $R$-point instability (except possibly in some of those systems for which we do not trust our potentials). In chalcogen-based systems this is not the case; ferroelectricity competes with and overwhelms the $R$-point instability. What remains a partially open question is whether this is simply due to the "sizes" of the crystal-field stabilized chalcogen ions, relative to those of the other constituent ions, being peculiarly favorable to ferroelectric behavior, or whether there is something unique about the chalcogen-metal bond that induces this behavior. If the former is the case, then ferroelectricity should presumably emerge from the present type of approach applied to these systems, and no new physical phenomenon is involved in its description. Its large degree of absence for halide-based systems is simply due to unfavorable ionic sizes: particularly those of the halide ions, as evidenced by the strengths of the ferroelastic distortions. If the latter is the case, as seems to us more likely, then the present theory has to be extended to incorporate crystal-field effects on the ionic charge densities in a self-consistent manner.

It is as well to conclude with a reemphasis of our initial caveat: The present theory predicates the perovskite structure and then examines its stability against (relatively) small distortions; we cannot rule out the existence of more absolutely stable structures involving very radical ionic rearrangements.

An additional caveat is also in order: We have not absolutely ruled out the possibility of a limited range of stability for a combined ferroelectric-ferroelastic phase in systems which show both $\Gamma$ - and $R$-point instabilities. This could arise, if, after a single $R$-point rotation had "frozen-in," there was a limited temperature range over which the system could further lower its free energy by transforming to a polar phase ( $\Gamma$-point distortion) before the final triple rotation occurs. On the basis of the evidence from the three systems we have examined in detail, this appears highly unlikely, these three will probably only be stable with the triple rotation frozen-in. For the other systems for which we trust our potentials, that we have not examined in the same detail, we think it to be unlikely, but not impossible. What is required is that the $R$ point instability be sufficiently weak for the phase with a single $R$-point distortion to be thermodynamically stable over a range of temperature, while the $\Gamma$-point instability must be sufficiently strong that the system can lower its free energy more easily by this distortion than by the triple rotation. These criteria appear to be mutually exclusive, since Tables I-III appear to indicate that a $\Gamma$ point instability is always "bought" at the expense of a strong $R$-point instability and, where present, does not itself "buy" much lowering of energy. A less unlikely possibility is that some systems may exist for which a single $R$-point rotation freezes-in, and which have a broad "double-well" potential for the $\Gamma$-point motion with associated low-frequency dielectric anomalies. A full resolution of this question would require a systematic study of free energies; a much more lengthy and complex investigation. However, one can make some estimates based on comparing the static energy differences for the three compounds we have studied in detail with those for $\mathrm{RbCaF}_{3}$, which we have previously studied, ${ }^{11}$ and for which we have also made approximate calculations for the present "triply rotated" minimum-energy state. The comparison does not look too favorable; the energy decreases for our present three systems are an order of magnitude larger than those for $\mathrm{RbCaF}_{3}$ making it very possible, as we pointed out earlier, that these systems would solidify in the triply rotated phase.

\section{ACKNOWLEDGMENT}

Support of this work at the University of Nebraska by the Office of Naval Research under Contract No. N00014-80-C-0518 is gratefully acknowledged. 
${ }^{1}$ F. A. Modine, F. Sonder, W. P. Unruh, C. B. Finch, and R. D. Westbrook, Phys. Rev. B 10, 1623 (1974).

${ }^{2}$ M. Rousseau, J. Y. Gesland, J. Julliard, J. Nouet, J. Zarembowitch, and A. Zarembowitch, Phys. Rev. B 12, 1579 (1975).

${ }^{3}$ C. Ridou, M. Rousseau, and A. Freund, J. Phys. (Paris) Lett. 38, L359 (1977).

${ }^{4}$ C. Ridou, M. Rousseau, J. Y. Gesland, J. Nouet, and A. Zarembowitch, Ferroelectrics 12, 199 (1976).

${ }^{5}$ H. Jex, J. Maetz, and M. Mullner, Phys. Rev. B 21, 1209 (1980).

${ }^{6}$ A. Bulou, C. Ridou, M. Rousseau, J. Nouet, and F. J. Shafer, J. Phys. (Paris) 41, 87 (1980).

${ }^{7}$ A. Bulou, J. Nouet, A. W. Hewat, and F. J. Shafer, Ferroelectrics 25, 375 (1980).

${ }^{8}$ C. Ridou, M. Rousseu, and J. Nouet, Ferroelectrics 26, 685 (1980).

${ }^{9}$ L. L. Boyer, Phys. Rev. Lett. 42, 584 (1979); Phys. Rev. B 23, 3673 (1981).

${ }^{10}$ L. L. Boyer, Phys. Rev. Lett. 45, 1858 (1980); 46, 1172 (1981).
${ }^{11}$ L. L. Boyer and J. R. Hardy, Phys. Rev. B 24, 2527 (1981).

${ }^{12}$ L. L. Boyer, Ferroelectrics 35, 83 (1981).

${ }^{13}$ L. L. Boyer, J. Phys. C (London) 17, 1825 (1984).

${ }^{14}$ M. J. Mehl and L. L. Boyer, Bull. Am. Phys. Soc. 29, 466 (1984).

${ }^{15}$ L. Pauling, The Nature of the Chemical Bond (Cornell University Press, Ithaca, 1946), p. 346.

${ }^{16}$ H. Arend, P. Muralt, S. Plesko, and D. Altermatt, Ferroelectrics 24, 297 (1980), and references therein.

${ }^{17}$ R. G. Gordon and Y. S. Kim, J. Chem. Phys. 56, 3122 (1971).

${ }^{18} \mathrm{E}$. Clementi and C. Roetti, Atomic Data and Nuclear Tables (Academic, New York, 1974).

${ }^{19}$ P. P. Ewald, Ann. Phys. 64, 253 (1921).

${ }^{20}$ There is experimental evidence [J. B. Bates, R. W. Major, and F. A. Modine, Solid State Commun. 17, 1347 (1975); W. A. Kamitakahara and C. A. Rotter, Solid State Commun. 17, 1350 (1975)] that a transition of this kind takes place in $\mathrm{RbCaF}_{3}$ at very low temperature $(\sim 50 \mathrm{~K})$. 